\title{
News from the Editor-in-Chief: impact factor 2010
}

\section{Domenico Prisco}

Published online: 12 July 2011

(C) SIMI 2011

Dear Readers,

Internal and Emergency Medicine (IEM) has obtained its second impact factor (IF), with a respectable 2.139 for 2010. IEM has received 154 citations of articles published in 2008 and 2009. After the impressive entry rank of 2.371 last year, the value of 2.139 is absolutely acceptable in light of IEM young age and of the increase of annual issues from 4 to 6 per year starting from 2009 with an increase in the number of published articles.

Our Journal is ranked 34th out of 151 journals in the category Medicine General and Internal. Last year, it ranked 30th out of 132 .

We are happy because our journal has been established as an internationally renowned in our academic field.

I wish to thank the Co-Editor, the Associate Editors and all our co-workers together with the management of Springer for this success and we hope to increase the IF in the future years.
During the first 6 months of 2011 we handled about 300 new submissions (vs. about 120 in the same period of 2010). The quality of submitted material is continuously increasing. The rejection rate is about $80 \%$ at the moment, due to this tremendous increase in the number of submitted papers.

Often medium-high quality papers are rejected only on the basis of non-high priority. We regret that some valuable colleagues are disappointed after our decisions, but we need to be more selective than in the past years.

We thank you, our Readers, and hope you will continue to support our Journal.

Domienico Prisco

Editor-in-Chief

Internal and Emergency Medicine

Conflict of interest None.

D. Prisco $(\bowtie)$

Dipartimento di Area Critica Medico Chirurgica,

Università degli Studi di Firenze,

Viale Morgagni 85, 50134 Florence, Italy

e-mail: priscod@aou-careggi.toscana.it 